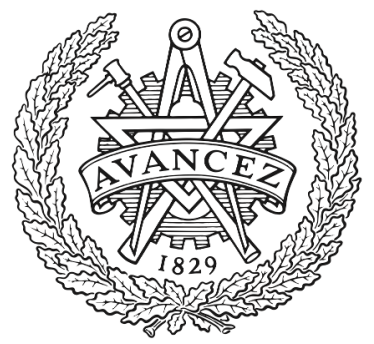

CHALMERS

UNIVERSITY OF TECHNOLOGY

\title{
Combining in Silico Tools with Multicriteria Analysis for Alternatives Assessment of Hazardous Chemicals: Accounting for the Transformation
}

Downloaded from: https://research.chalmers.se, 2023-04-26 08:15 UTC

Citation for the original published paper (version of record):

Zheng, Z., Arp, H., Peters, G. et al (2021). Combining in Silico Tools with Multicriteria Analysis for Alternatives Assessment of Hazardous

Chemicals: Accounting for the Transformation Products of decaBDE and Its Alternatives.

Environmental Science and Technology, 55(2): 1088-1098. http://dx.doi.org/10.1021/acs.est.0c02593

N.B. When citing this work, cite the original published paper. 


\title{
Combining In Silico Tools with Multicriteria Analysis for Alternatives Assessment of Hazardous Chemicals: Accounting for the Transformation Products of decaBDE and Its Alternatives
}

\author{
Ziye Zheng,* Hans Peter H. Arp, Gregory Peters, and Patrik L. Andersson
}

Cite This: Environ. Sci. Technol. 2021, 55, 1088-1098

Read Online

\section{ACCESS |}

Џll Metrics \& More

回国 Article Recommendations

Supporting Information

ABSTRACT: Transformation products ought to be an important consideration in chemical alternatives assessment. In this study, a recently established hazard ranking tool for alternatives assessment based on in silico data and multicriteria decision analysis (MCDA) methods was further developed to include chemical transformation products. Decabromodiphenyl ether (decaBDE) and five proposed alternatives were selected as case chemicals; biotic and abiotic transformation reactions were considered using five in silico tools. A

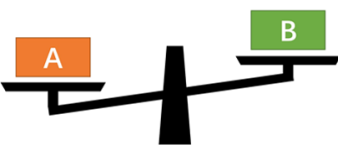

Parent compounds

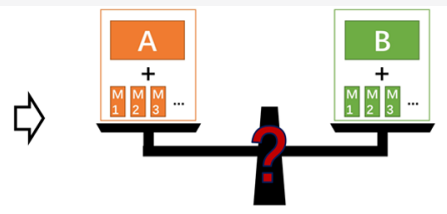

Parent compounds and transformation products (M1, M2...) workflow was developed to select transformation products with the highest occurrence potential. The most probable transformation products of the alternative chemicals were often similarly persistent but more mobile in aquatic environments, which implies an increasing exposure potential. When persistence $(\mathrm{P})$, bioaccumulation (B), mobility in the aquatic environment $(\mathrm{M})$, and toxicity $(\mathrm{T})$ are considered (via PBT, PMT, or PBMT composite scoring), all six flame retardants have at least one transformation product that can be considered more hazardous, across diverse MCDA. Even when considering transformation products, the considered alternatives remain less hazardous than decaBDE, though the range of hazard of the five alternatives was reduced. The least hazardous of the considered alternatives were melamine and bis(2-ethylhexyl)tetrabromophthalate. This developed tool could be integrated within holistic alternatives assessments considering use and life cycle impacts or additionally prioritizing transformation products within (bio)monitoring screening studies.

\section{INTRODUCTION}

Transformation of chemicals is an important but often neglected issue in chemical risk assessment. The toxicological risks associated with chemicals can be underestimated if only parent compounds are considered, as some chemicals can transform into more hazardous compounds. For example, the dry cleaning solvent perchloroethylene, which was widely used before the 1990s, can reductively degrade to the more toxic vinyl chloride. ${ }^{1}$ Bronopol (2-bromo-2-nitro-1,3-propanediol), which has been used as a preservative in pharmaceutical and personal care products, can undergo transformations to the persistent and toxic products 2-bromo-2-nitroethanol and bromonitromethane in aquatic environments. ${ }^{2}$ Carbamazepine, an antiepileptic pharmaceutical agent, has been shown to photodegrade into the more toxic products acridine and acridone.

Chemical regulation and risk assessment guidelines have been created to address the importance of including transformation products. For example, the European chemical regulation Registration, Evaluation, Authorisation and Restriction of Chemicals (REACH) emphasizes that transformation products should be included in the persistence, bioaccumulation, and toxicity (PBT) assessment. ${ }^{4}$ The importance of considering transformation products in risk assessment has been an ongoing topic of discussion for several substances, ${ }^{5,6}$ such as for the flame retardant decabromodiphenyl ether (decaBDE). ${ }^{7,8}$ Although decaBDE itself is considered to have low bioaccumulation potential and low toxicity, it can undergo environmental transformation reactions into dozens of other polybromodiphenyl ethers (PBDEs) that are more bioaccumulative and toxic than decaBDE; therefore, it was evaluated under REACH as a PBT substance.,

Alternatives assessment frameworks assess substances with the same or similar uses considering both environmental and human health aspects, including life cycle impacts, product performance, technical suitability, cost, and social responsibility, to minimize the risk of regrettable substitutions. ${ }^{9,10}$ The importance of including transformation products is often pointed out in chemical hazard assessments used in alternatives assessment frameworks like GreenScreen, ${ }^{11}$ U.S. Environmental Protection Agency (EPA) Design for Environment (DfE) Program, ${ }^{12}$ and recently by Martin. ${ }^{13}$ However, this remains a difficult task, as many of the chemicals considered in

Received: April 24, 2020

Revised: December 14, 2020

Accepted: December 15, 2020

Published: December 31, 2020 
alternatives assessments are novel compounds that have been subjected to a few experimental investigations themselves. Experimental data on their transformation products are even more scarce. Therefore, using recommended experimental approaches to identify transformation products that pose the most risk, such as combining exposure assessment with effectdriven assessments using ecotoxicity assays, ${ }^{14}$ is not generally an option at the initial, screening stage. In such cases, in silico tools can be useful to predict transformation products as well as their hazardous properties. For example, GreenScreen ${ }^{11}$ suggests the use of models provided by the OECD QSAR Toolbox ${ }^{15}$ for predicting chemical transformation when there are no experimental data available. Martin ${ }^{13}$ used the Chemical Transformation Simulator (CTS $)^{16,17}$ provided by USEPA to predict biotransformation products for case chemicals. There is a range of other in silico tools available that have been used for the prediction of transformations products for chemical risk assessment of organic chemicals, including commercial tools such as Metasite, ${ }^{18}$ Stardrop, ${ }^{19}$ Zeneth, ${ }^{20}$ and Meteor Nexus, ${ }^{21}$ as well as open-source tools such as BioTransformer ${ }^{22}$ and the EAWAG-biocatalysis/biodegradation database pathway prediction system (EAWAG-BBD/PPS). ${ }^{23}$ A key challenge when using such tools is a meaningful interpretation of their output. They typically generate a large number of different transformation products, especially if the software includes a large variety of transformation pathways. Even if estimations of the relative fraction of the products formed or the likelihood of formation are provided by the software, they are always associated with some uncertainty. Further, even if some experimental data are available, it must be considered that the transformation rate as well as exposure level for each product are hard to measure since they are highly dependent on environmental conditions (e.g., temperature, microbial communities, $\mathrm{pH}$ ) and exposure routes (e.g., sludge, soil, atmospheric, and aquatic). ${ }^{13,24}$ These challenges all contribute to there being very few published alternatives assessment case studies that take transformation products into consideration. One exception to this was Martin, ${ }^{13}$ which concerns decaBDE as well as three proposed organophosphate-based alternative flame retardants. In that study, transformation products predicted by $\mathrm{CTS}^{16,17}$ were considered and hazard information was given for each of the parent compounds and transformation products, though without providing a hazard ranking for alternative selection. Herein, we wish to expand on such a study by considering a broader array of in silico tools, flame retardants, hazard criteria, and hazard ranking approaches via applying multiple multicriteria decision analyses (MCDA) to assist in evaluating this larger dataset. The use of MCDA methods has become increasingly popular in the field of alternatives assessment, as these methods can be used not only for drawing more trustworthy conclusions but also for identifying the most critical criteria. ${ }^{25}$

In our previous study, ${ }^{26}$ an efficient hazard ranking tool was established for alternatives assessment by combining opensource quantitative structure-activity relationship (QSAR) model hazard data and MCDA methods with the consideration of data uncertainties. Herein, we extend this hazard ranking tool to include and select important transformation products predicted by in silico tools and use multiple MCDA methods for a joint hazard ranking. The specific aims are to: (a) use available in silico tools, especially the open-source ones, to predict transformation products of the case chemicals to see the availability and any limitations of these tools; (b) develop a strategy to select the most important transformation products from all available tools to reduce uncertainty, and validate with experimental data; (c) adapt the MCDA methods used in the previous study to include transformation products; and finally (d) see if the hazard ranking of alternative flame retardants changes when transformation products are considered.

\section{MATERIALS AND METHODS}

Case Chemical Selection and Hazard Data Calculation. Our previous study ${ }^{26}$ derived hazard ranking results of decaBDE and 16 possible alternative flame retardants using different MCDA methods. This was mainly done using in silico data, as available experimental data were not sufficient for a complete assessment of all hazard endpoints of each alternative flame retardant (though, in general, high-quality experimental data are favored when available). Four hazard properties including persistence $(\mathrm{P})$, bioaccumulation $(\mathrm{B})$, mobility in water $(M)$, and toxicity $(T)$ were considered, and hazard ranking was done using three MCDA methods: heat mapping, multiattribute utility theory (MAUT), and Elimination Et Choix Traduisant la Realité (ELECTRE III). In the present study, decaBDE, which was identified among the most hazardous chemicals in the previous study, ${ }^{26}$ was selected with the three chemicals that were evaluated to have the relatively lowest hazard; decabromodiphenyl ethane (DBDPE), bis(2-ethylhexyl) tetrabromophthalate (BEHTEBP), and melamine (MA). Additionally, organophosphate flame retardants (OPFRs) were considered, even though they were not amongst the least hazardous alternatives, because they are an important class of flame retardants considered in a similar study. ${ }^{13}$ Here two OPFRs were included to represent halogenated and nonhalogenated OPFRs, respectively: tris(tribromoneopentyl) phosphate (TTBNPP) and triphenyl phosphate (TPHP). Collectively, these alternatives cover a broad array of hazard criteria and substance classes: brominated flame retardants, brominated OPFRs, halogenfree OPFRs, and melamine. Chemical information for these six case chemicals is shown in Table $S 1$ in the Supporting Information (SI).

Hazard data were calculated using the same models as in the previous study for both the six flame retardants and their transformation products. $^{26}$ In brief, chemical structure information (SMILES) was used to derive data from 55 QSAR models including models from the open-source tools EPISUITE, ${ }^{27}$ VEGA, ${ }^{28}$ TEST, $^{29}$ and OECD QSAR Toolbox; ${ }^{15}$ models for endocrine related responses on the OCHEM platform ${ }^{30}$ from the USEPA organized Collaborative Estrogen Receptor Activity Prediction Project (CERAPP), ${ }^{31}$ Collaborative Modelling Project for Androgen Receptor Activity (CoMPARA), ${ }^{32}$ and the literature ${ }^{33}$ (Table S2). These models covered 20 hazard criteria for $\mathrm{P}, \mathrm{B}, \mathrm{M}$, and $\mathrm{T}$ (Table S2). It is noted that for the selection of QSARs, it was an intentional focus to use open-source/access models that can easily be applied by potential users. ${ }^{26}$

Decision Analysis. Three MCDA methods were used for hazard ranking including heat mapping, MAUT, and ELECTRE III. Thresholds for all three MCDA methods are presented in Table S3. For the heat map, the range of each criterion was divided into four color-coded intervals (green $=$ benign, yellow $=$ moderate hazard, orange $=$ high hazard, red $=$ very high hazard). For the MAUT approach, each hazard criterion was scaled from 0 (worst) to 1 (best) based on the distance between the hazard level of target flame retardants to 
Table 1. Overview of In Silico Tools Used for Transformation Product Prediction, Presenting the Transformation Pathways, Number of Transformation Steps, and Probability Ranking System Included in Each

\begin{tabular}{|c|c|c|c|c|}
\hline process & software & transformation pathways & $\begin{array}{l}\text { stepwise transformation } \\
\text { predictions }\end{array}$ & probability ranking \\
\hline \multirow[t]{4}{*}{$\begin{array}{l}\text { mammalian } \\
\text { metabolism }\end{array}$} & Meteor Nexus & $\begin{array}{l}\text { phase I and phase II metabolism for mammals } \\
\text { (human, dog, and rat) }\end{array}$ & $\begin{array}{l}\text { yes, with a limitation of maximum } \\
5000 \text { metabolites }\end{array}$ & $\begin{array}{l}\text { one classification ranking and } \\
\text { two scoring methods }\end{array}$ \\
\hline & CTS & phase I metabolism for human & yes, up to four steps & classification ranking \\
\hline & QSAR Toolbox & phase I and phase II metabolism for rat & no & not provided \\
\hline & BioTransformer & phase I and phase II metabolism for mammals & only one step at a time & not provided \\
\hline \multirow[t]{3}{*}{$\begin{array}{l}\text { microbial } \\
\text { metabolism }\end{array}$} & QSAR Toolbox & $\begin{array}{l}\text { environmental and biotic microbial } \\
\text { transformations }\end{array}$ & no & not provided \\
\hline & $\begin{array}{l}\text { EAWAG-BBD/ } \\
\text { PPS }\end{array}$ & & yes & classification ranking \\
\hline & BioTransformer & & only one step at a time & not provided \\
\hline \multirow{2}{*}{$\begin{array}{l}\text { abiotic } \\
\text { transformation }\end{array}$} & CTS & hydrolysis and reduction & yes, up to four steps & classification ranking \\
\hline & QSAR Toolbox & autoxidation, dissociation, and hydrolysis & no & not provided \\
\hline
\end{tabular}

a set level for this criterion (further explained in SI). As pointed out by the previous study, ${ }^{26}$ chemical applicability domain and prediction quality data of the QSAR models vary $^{34-36}$ and are not transparent or in some cases available for the diverse range of in silico tools used for hazard predictions. Comparisons between outputs of different models for the same hazard criteria as well as modeling outputs with experimental data suggest that there can be substantial prediction uncertainties. Therefore, for ELECTRE III, pairwise comparisons were conducted for target chemicals on each hazard criterion, and the significant difference levels between chemicals for each criterion (i.e., the indifference thresholds and preference thresholds in ELECTRE III) were set based on standard deviations of different model results for the same criteria to consider model uncertainties (see the SI). In this study, thresholds were kept from the previous study as the same in silico tools were used. For both MAUT and ELECTRE III, each hazard criterion for $\mathrm{P}, \mathrm{B}, \mathrm{T}$, or $\mathrm{M}$ properties was assigned the same weight, and final scores were calculated by treating the composite criteria of PBT, PMT, or PBMT as equally important (referred to as PBT, PMT, or PBMT score). Further explanation of MAUT and ELECTRE III are presented in the SI. In this study, these three decision methods were used to compare the hazard of transformation products with their parent compounds. In addition, they were used for an overall alternatives assessment including both parent and potential transformation products.

Predictions of Transformation Products. To cover different transformation pathways and to compare the predictive abilities of different in silico tools, several opensource software platforms were considered: OECD QSAR Toolbox, ${ }^{15}$ CTS, ${ }^{16,17}$ BioTransformer, ${ }^{22}$ and EAWAG-BBD/ PPS. ${ }^{23}$ In addition, one commercial software package, Meteor Nexus, ${ }^{21}$ was included as a complement including both a rulebased expert system providing a classification rank from "improbable" to "probable" for each transformation product and machine learning methods that yield probability scoring for different products. The open-source tools provide either a likelihood of transformation reactions (CTS and EAWAG$\mathrm{BBD} / \mathrm{PPS}$ ) or only indicate whether the product is formed (no ranking) (QSAR Toolbox and BioTransformer). The selected tools cover a wide range of transformation pathways, where QSAR Toolbox, CTS, BioTransformer, and Meteor can predict mammalian metabolism for different species; QSAR Toolbox, EAWAG-BBD/PPS, and BioTransformer include predictions for microbial metabolisms; and CTS and QSAR Toolbox also cover some abiotic transformation pathways (Table 1). Photodegradation is not covered by the selected tools, though there have been some advances to predict photodegradation. ${ }^{37}$

Selection of Predicted Transformation Products. An important limitation for all used in silico tools for the prediction of transformation products is that there is no obvious information regarding the modeling applicability domain. It is therefore hard to evaluate the quality of predictions. To address this, we developed a strategy using in silico data to prioritize predicted transformation products that have the highest probability of occurrence, i.e., the highest occurrence potential, and that exhibit a similar or greater intrinsic hazard compared to the parent compound. This was inspired by similar approaches for selecting important transformation products by previous studies. ${ }^{14,38-41}$

One such approach was the framework by Escher and Fenner ${ }^{14}$ to prioritize transformation products that meet both an exposure-based threshold (via fractions of formation through relevant transformation pathways) and toxicity-based threshold compared to the parent structure. Another key approach was $\mathrm{Ng}$ et al., ${ }^{38}$ who developed an in silico framework to select key transformation pathways (with EAWAG-BBD) and prioritize products with the most persistence and exposure potential compared to the parent (with EPISuite and a multimedia fate model). The in silico framework we develop here shares similar elements with both of these approaches but expands and deviates on many aspects. Here, we deploy an array of transformation models, not just EAWAG-BBD, to prioritize the transformation products for consideration by cross-validation; and instead of looking at exposure via a multimedia approach as in $\mathrm{Ng}$ et al., ${ }^{42}$ we assess the P, B, and $\mathrm{M}$ criteria separately to be more consistent with the current REACH framework. Finally, we additionally consider toxicity $(T)$ as in Escher and Fenner, ${ }^{14}$ as a basis for selecting transformation products.

Selecting compounds with highest occurrence potential is not straightforward since transformation rates are not given by the in silico tools, and these rates are anyway dependent on environmental conditions and pathways. To address these 
concerns, herein, we defined the transformation products with highest occurrence potential based on the following criteria: (i) they are predicted to occur through the greatest number of in silico pathways/models, i.e., have a high occurrence frequency across models, and (ii) they have a high predicted persistency. The reason for the first condition is in part a quality control check. When the different models, different pathways, and different calibration datasets lead to the same transformation reactions and/or products, at least some cross-model validation is provided. The reason for the second condition is that transformation products with high persistence can accumulate over time and potentially cause high exposure depending on the fraction of formation. ${ }^{38,43}$ Based on these assumptions, a workflow was developed for prioritization of potential transformation products, as shown in Figure 1.

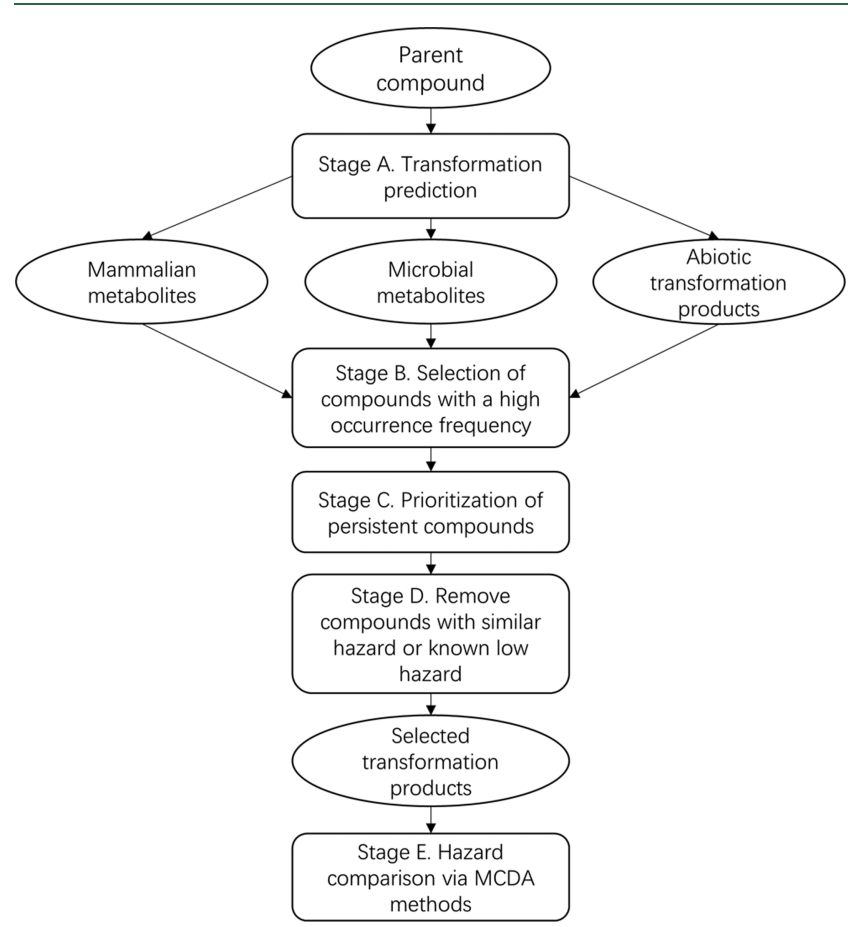

Figure 1. Workflow for the prioritization of predicted transformation products based on the highest occurrence potential (stages B and C combined) and hazard (stages D and E).

To address stage B (Figure 1) regarding a high occurrence frequency, the following strategy was implemented; the first generation of transformation products is given primary focus, but the second, third, and subsequent generations are considered under specific circumstances. The specific rules for stage $B$ are:

- For mammalian metabolism, all first-step products from phase I or phase II metabolism that were predicted by more than one in silico tool are included; for second and third steps, metabolic products, i.e., only metabolism products from reactions that are both predicted "likely" (all the way from parent compound to the target metabolites) by CTS (which marks the likelihood of reactions as unlikely, probable, and likely) and given a site of metabolism (SOM) score of more than 300 by Meteor Nexus are included. Meteor Nexus has three methods for probability ranking of metabolites, wherein
SOM was reported elsewhere to be the most accurate one $^{44}$ and thus chosen for this study.

- For microbial metabolism, BioTransformer and EAWAG-BBD/PPS give very similar results since BioTransformer adopted the EAWAG-BBD biotransformation rule library. Products identified by both QSAR Toolbox and EAWAG-BBD/PPS (which marks the likelihood of reactions as unlikely, neutral, likely, and very likely) with a probability of reactions not lower than neutral (all the way from parent compound to the target metabolites) within three steps were included.

- For abiotic transformation, products identified by CTS with a predicted probability of reactions considered likely (all the way from parent compound to the target metabolites) and by QSAR Toolbox within three steps were included.

For stage $\mathrm{C}$ in Figure 1, prioritization based on persistence, only compounds with at least a moderate persistence level in sediment were considered. The cutoff values for moderate persistence level were taken from the heat mapping in a previous study ${ }^{26}$ based on regulation and literature levels (Table S3). The reason for using sediment as the environmental compartment for $\mathrm{P}$ is that half-lives predicted in EPISuite for water, soil, and sediment are proportional to each other with a ratio 1:2:9 as they are all derived from the same BIOWIN output within EPISuite, and therefore predictions for sediment are the most conservative. This type of extrapolation is one of the many uncertainties associated with half-life predictions using EPISuite. ${ }^{45}$ However, since EPISuite remains the most common, open-source predictor of half-lives, these models were used in this study for hazard assessment as in our previous study.

Finally, regarding step D in Figure 1 on the initial hazard evaluation, only one structure example was selected for each considered homologue group of the PBDEs, hydroxylated PBDEs, and bromodiphenyl ethanes, i.e., one structure per number of bromine substituents (e.g., one tribrominated, one tetrabrominated, etc., selected structure examples are presented in Table S5) and small compounds with a benign hazard (e.g., $\mathrm{NH}_{3}, \mathrm{CO}_{2}$ ) were excluded.

\section{RESULTS AND DISCUSSION}

Transformation Products Predicted by In Silico Tools. The number of transformation products of the case chemicals that were predicted by the selected tools for the various pathways are presented in Table S4. An illustrative example of such outcomes for all substances, and how such data were interpreted following the workflow in Figure 1, is presented in Figure 2. This figure shows the predicted results of three consecutive steps of mammalian metabolites of melamine predicted by BioTransformer, CTS, and Meteor Nexus (QSAR Toolbox identified no metabolites). Since BioTransformer can predict only one generation at a time, all predicted phase I products were used as input again until no further products were predicted, or the third generation was reached. The phase I transformation products are quite similar for CTS and Meteor Nexus, while BioTransformer appeared to deliver fewer predicted compounds. In average, $31 \%$ of the first-step mammalian metabolites were predicted by more than one in silico tool and $6 \%$ by all available tools (Table S4). However, for some chemicals, the output differed widely between the tools. For example, Meteor Nexus predicted many trans- 


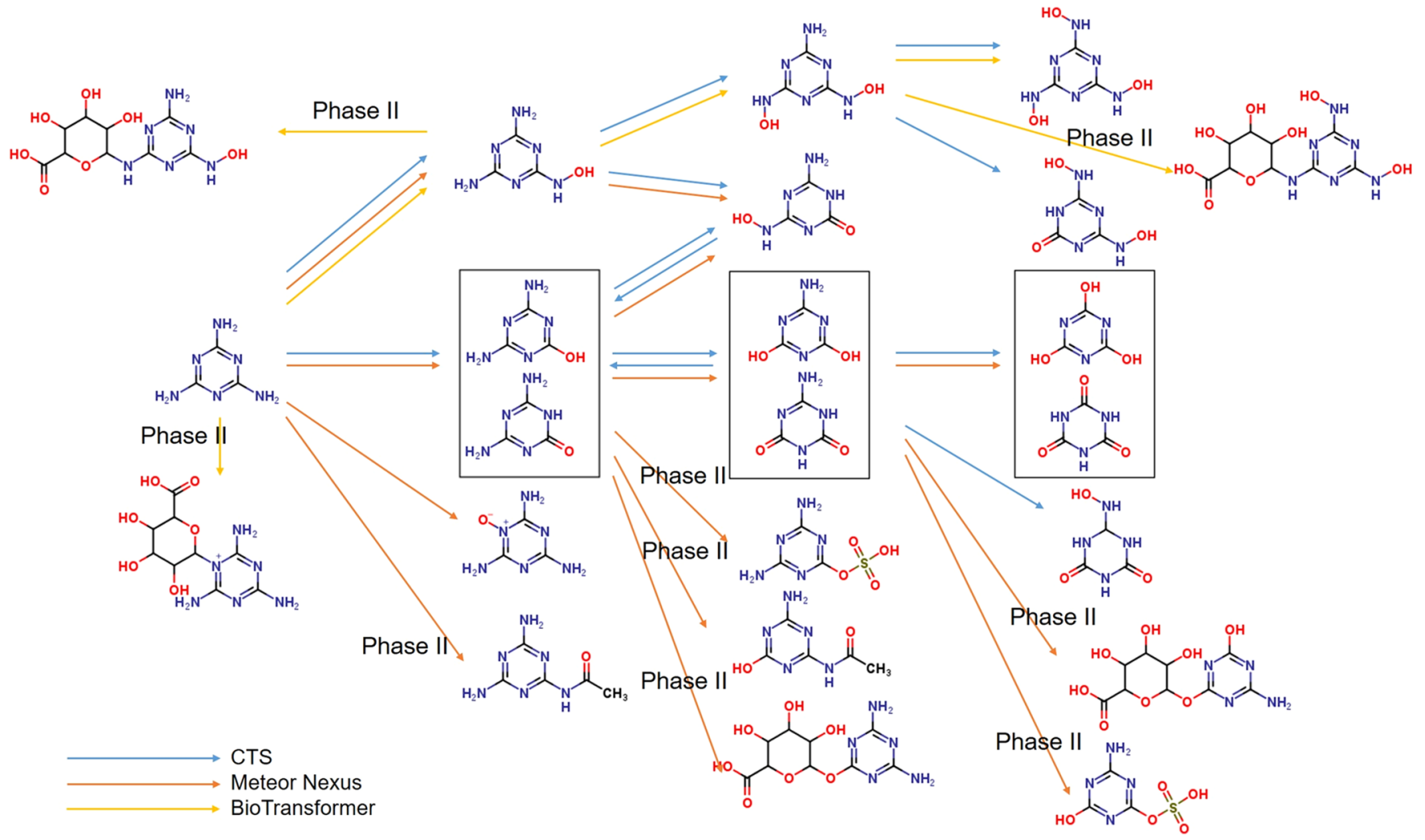

Figure 2. Mammalian metabolites of melamine predicted by different in silico tools. Structures given in boxes are tautomers.

formation products for decaBDE, DBDPE, and TTBNPP, including 300-400 unique transformation products for the first three steps of mammalian metabolism for each of these three flame retardants (Table S4), while the other tools predicted no or very few mammalian metabolites. The large number of metabolites predicted by Meteor Nexus is caused by the parent compound being predicted to be very persistent, making the SOM algorithm give all metabolites a relative score " 0 " and preventing proper ranking of the metabolites. Therefore, according to the rules presented above in relation to Figure 1, the output of Meteor Nexus for decaBDE, DBDPE, and TTBNPP was excluded. For the other three compounds (BEH-TEBP, TPHP, and TTBNPP), the numbers of transformation products predicted by Meteor Nexus and CTS are closer (Table S4). For phase II transformations, Meteor Nexus predicted more phase II transformation products compared to other software, as evident in Figure 2 for melamine. Since the open-access tools are not as transparent regarding which pathways/enzymes were included and how probability was assessed compared with Meteor Nexus, it is difficult to determine the exact reason for the differences between the predictions of the different tools. The outcomes of the microbial metabolism predictions were similar in that the selected tools gave similar results for some target chemicals and gave disparate results for the others. For example, 112 microbial metabolites of DBDPE were predicted by QSAR Toolbox, while EAWAG-BBD/PPS only predicted 4. Examples for other substances can be found in Table S4.

A number of different transformation mechanisms were predicted for the case chemicals. Step-by-step oxidation, hydroxylation, and debromination of polybrominated compounds are the most frequently observed reactions for phase I transformations. More complex reactions such as ring cleavage, e.g., from cyanuric acid (from melamine, MA-M4) to biuret and allophanate (MA-M5 and MA-M6), were more commonly encountered in microbial transformation simulations than mammalian or abiotic. Abiotic transformation simulations are composed exclusively of autoxidation, dissociation, reductive debromination, and hydrolysis mechanisms.

Persistence Evaluation. The outcomes of the workflow in Figure 1 to prioritize predicted transformation products are listed in Table S5. The number of prioritized transformation products ranged from five to nine for each of the case chemicals. Each of the melamine transformation products ammeline, ammelide, and cyanuric acid has two tautomer structures (alcohol and ketone), and both structures are included in this study (MA-M2, M2', M3, M3', M4, and M4'). Interestingly, none of the transformation products selected by stage B (occurrence frequency) were ruled out by stage C (persistence), as the predicted half-lives in sediment are all beyond the moderate persistence limitation that was used here as a cutoff for persistence (Table S3). This indicates that many transformation products of persistent compounds are also likely to be persistent. According to the BIOWIN results, this is due to stable fragments within the parent substances (carbonbromine bonds, highly aromatic triazine structures, etc.) being generally retained in the transformation products. Also, the high molecular weights of some transformation products contributed to a similar P estimation.

Comparison with Experimental Data. Most of the experimentally identified transformation products for decaBDE and melamine available in the literature (Table S6) were identified by the in silico tools and prioritized by the workflow. For example, nonaBDEs, octaBDEs, hydroxylated nonaBDE, and hydroxylated octaBDEs are formed from decaBDE and were predicted by the in silico tools. ${ }^{46-49}$ Ammeline, ammelide, 


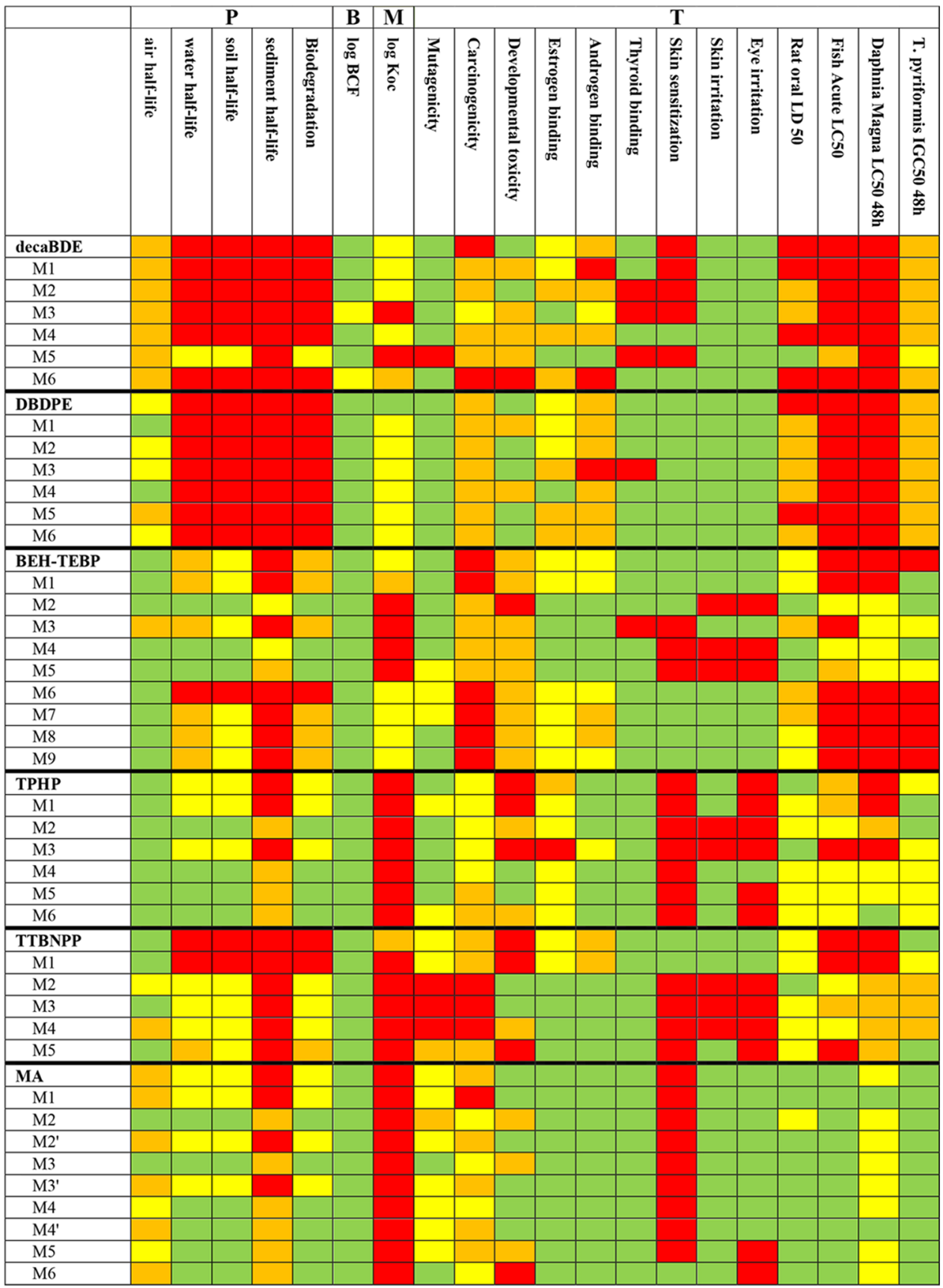

Figure 3. Heat map of the six flame retardants and their selected transformation products, where red indicates that a hazard criterion has been met, orange and yellow indicate high and moderate hazard levels, respectively, though below the cutoff for the red level, and green indicates that the chemical has properties that fulfilled a set safe level. The levels were set based on regulations and literature values according to our previous study. ${ }^{26}$ Metabolites marked with and without an apostrophe, e.g., M4 and M4', refer to tautomeric forms.

and cyanuric acid have been identified experimentally as transformation products of melamine $e^{42,50,51}$ and were also prioritized via the in silico approach here. The most widely identified transformation product for TPHP, diphenylphosphate, ${ }^{52-56}$ and the only studied transformation product for BEH-TEBP, mono(2-ethyhexyl) tetrabromophthalate ${ }^{57}$ (Table S6), were also predicted by the in silico tools and identified as having high occurrence potential. This gives some validation of the appropriateness of our tools and presented workflow for hypothesizing transformation products with a relevant occurrence potential.
However, in addition to these matches, there were a large number of predicted transformation products that could not be found in the literature, even for decaBDE and melamine. At face value, these in silico tools are overpredicting based on the available experimental data and are likely introducing false positives due to low selectivity precision in pathway prediction, ${ }^{58}$ but this cannot be formally evaluated for these test compounds in this study due to limited simulation and analytical data.

There are some metabolites in the literature that were not predicted, which could be considered false negatives, but these are exceptional cases or of low fractions of formation. Notably, 
methoxylated PBDEs were identified as metabolites of decaBDE in rats, ${ }^{46-48}$ while they were not identified by any of the applied in silico tools. Some additional transformation products, for example, PBDEs with three to seven bromines for decaBDE, ${ }^{49,59-62}$ and biuret and allophanate as ring cleavage products of melamine, ${ }^{42,50}$ have been identified in some experimental studies, though at relatively low concentrations (Table S6). These compounds are degradation products beyond the first three transformation steps and are either not identified by the in silico tools or not prioritized by our approach since it focuses on transformation products with high occurrence potential. Pentabromodiphenyl ethers (pentaBDE) did not have a high occurrence potential through our approach as a transformation product for decaBDE, despite this being a major consideration in decaBDE hazard and risk assessments. ${ }^{8,13}$ Part of the explanation for this is the lack of predictive tools for photodegradation since pentaBDE has been considered as an important photodegradation product of decaBDE ${ }^{7,8,61,62}$ and also that this would be a transformation product after five subsequent debromination reactions. However, because pentaBDE is considered a critical transformation product of decaBDE, it was also included herein for further hazard assessment. Biuret and allophanate were also included as transformation products of melamine, as such experimental data should be integrated when available.

Hazard Comparison between Studied Flame Retardants and Their Transformation Products. A heat map was produced based on regulation or literature set levels for the various hazards under consideration (Figure 3). At least two red flags occurred for all compounds, including all selected transformation products. This implies that the major transformation products of these hazardous compounds are all hazardous in some way. Another heat map was produced to compare each case chemical with its selected transformation products (Figure S1). One intrinsic consideration here with heat maps is that that the longer the list of included hazard criteria, the more likely a red flag will occur, hence the need for MCDA methods in this context. Nevertheless, the heat maps are useful in rapidly comparing burden-shifting of hazards across parent compounds or their transformation products.

It can be seen from both figures that most of the selected transformation products have similar environmental persistence compared with the parent compounds ( 32 of the 41 selected transformation products were predicted to have no considerable difference, as defined by the veto threshold, in persistence in all environmental media; see Figure S1). The workflow (Figure 1) is biased toward this result, as the predicted transformation products have to meet the implemented cutoffs for persistency (stage $\mathrm{C}$ in Figure 1, i.e., predicted estimated sediment half-life $>60$ days). The transformation products for the polybrominated compounds (decaBDE, DBDPE, and TTBNPP) with a high occurrence frequency are persistent in the sediment with an estimated halflife of more than 180 days, and many of them are also persistent in biota with an estimated biodegradability half-life of more than 60 days.

Almost all transformation products are more mobile than their parent compounds. This is consistent with the outcome of a study that compared persistency and mobility of predicted hydrolysis products of all REACH registered substances with the parent substances, ${ }^{34}$ as well as an earlier study with pesticides. ${ }^{63}$ These studies reported overall increased mobility for transformation products, due to additional polar functional groups, but no substantial change in predicted persistence.

Some transformation products were also more bioaccumulative; these were products from decaBDE, DBDPE, and $\mathrm{BEH}$ TEBP, whereas others were less bioaccumulative (the two OPFRs) or showed no change (melamine). Note that B was assessed by bioconcentration factors (BCF), as suggested by $\mathrm{REACH}$, while as discussed in our previous study, dietary uptake might be of more importance for these hydrophobic chemicals; thus, bioaccumulation factors (BAF) might be a more appropriate choice. The only available BAF model was through EPISuite, and a comparison between BAF and average BCF shows that if BAF was used to create the heat map, most of the compounds would be marked as the same color except for DBDPE-M5; DBDPE-M6 and decaBDE-M2 would shift from green to yellow; decaBDE-M1 and decaBDE-M4 would shift from green to red; and decaBDE-M6 would shift from yellow to red (Table S7). As a result, changing BCF to BAF would have no substantial impact on the studied flame retardants except for possible worse ranking for decaBDE, which however already appeared to be the worst alternative. Also, the uncertainty for estimated BAF is overall higher as it only has one model. Consequently, later, MCDA approaches were based on BCF for the sake of consistency.

Accounting for changes in toxicity during transformation is complex; however, in general, burden-shifting was observed for all transformation products. All 45 identified transformation products are considerably worse than the parent compound for at least one toxicity criterion, and 42 of them are also considerably better than the parent compound for at least one toxicity criterion (Figure S1). The burden-shifting of toxicity for transformations was also observed by Martin. ${ }^{13}$ Adding to the complexity, toxicity data from animal studies could potentially be caused by the dosed parent compound or formed metabolites. Although the heat maps clearly show the burden-shifting between different kinds of toxicity, they are insufficient for making aggregated hazard rankings; thus ranking methods such as MAUT and ELECTRE III are required.

The outcome of MAUT was that five of the six target flame retardants (except for melamine) transformed into at least one compound that was ranked worse according to the PBT score, and all six transformed into at least one more hazardous compound according to the PBMT score (Table S10). Among the 41 selected transformation products for the six flame retardants, 13 of them were ranked more hazardous compared to their own parent compound for the PBT score and 24 of them were more hazardous for the PBMT score. The average MAUT score for PBMT of transformation products for each of the three brominated flame retardants is worse than their parent compound and, notably, all transformation products of DBDPE and BEH-TEBP are more hazardous than their parent compounds. The uncertainty in in silico hazard data needs to be taken into consideration in hazard ranking, as recently discussed by Muir et al.; ${ }^{36}$ thus, the MAUT results were examined by ELECTRE III, which considered the prediction uncertainty. ELECTRE III results generally agreed with MAUT (wherein 19 of the 41 transformation products ranked more hazardous than their parent compounds by PBT and 20 by PBMT; Tables S11-S22), indicating that the uncertainty in the in silico hazard data has generally low impact in this specific case study. This does not imply that this uncertainty is a minor issue in general; it just happened to be so here because the 
differences in the PBT and PBMT hazard scoring between the transformation products were generally similar to or larger than the uncertainty of the scoring.

A fundamental limitation for most in silico tools is that they were either not trained with ionizable compounds or even if they were (e.g., the KOCWIN fragment-based method module is trained with both neutral and ionized substances) they may not make explicit $\mathrm{p} K_{\mathrm{a}}$-based corrections for changes in ionization over an environmental $\mathrm{pH}$ range. This might, in particular, have an impact on the assessment of $\mathrm{P}, \mathrm{B}$, and $\mathrm{M}$, depending on the $\mathrm{p} K_{\mathrm{a}}$ value of the compound. Previous studies also suggest that EPISuite has poor predictions for B and $\mathrm{M}$ for ionic or ionizable compounds that dissociate within an environmental relevant $\mathrm{pH}$ range of $4-9 .{ }^{34,45}$ For the 47 studied compounds (both parent compounds and transformation products), based on the $\mathrm{p} K_{\mathrm{a}}$ values estimated by Chemaxon, 18 are not always in neutral form in the $\mathrm{pH}$ range of $4-9,{ }^{64}$ and 16 are not in neutral form at $\mathrm{pH} 7$ (Table S8). If the minimum octanol-water partition coefficient (logDow) estimated by Chemaxon within the $\mathrm{pH}$ range of 4-9 was used to assess mobility instead of the organic carbon-water partition coefficient of the neutral form $\left(\log K_{\mathrm{oc}}\right)$, BEHTEBP-M1 would shift from orange to red in the heat map, while eight compounds (mostly transformation products of DBDPE) would shift from yellow to green (Table S9). It is worth pointing out that Chemaxon is the only tool we applied for the estimation of $\mathrm{p} K_{\mathrm{a}}$ and $\log D_{\mathrm{ow}}$, and the uncertainty can be high for some compounds. ${ }^{34,35}$ For example, the $\mathrm{p} K_{\mathrm{b} 1}$ for the protonated form of melamine is estimated to be 9.5 , while the experimental measured value is 5.0. ${ }^{65} \mathrm{~A}$ needed advancement for this hazard ranking tool is to identify, develop, and further develop the tool to be suitable for ionic compounds.

Alternatives Assessment with Consideration of Transformation Products. Our previous study ${ }^{26}$ identified BEH-TEBP, melamine, and DBDPE as the least hazardous alternatives for decaBDE using both MAUT and ELECTRE III considering both PBT and PBMT. According to the PBMT ranking of the most hazardous transformation products, $\mathrm{BEH}$ TEBP, melamine, and DBDPE still retain their status of the least hazardous alternatives (Figure 4a). The two worst transformation products identified by MAUT (PBMT) for each flame retardant were assessed by ELECTRE III together with their parent compounds (i.e., for TPHP, TPHP-M3, TTBNPP, and TTBNPP-M1). ELECTRE III also considered BEH-TEBP and melamine preferable, whereas TTBNPP ranked better than with MAUT, and DBDPE ranked worse than with MAUT for both PBT and PBMT (Figure 4a and Tables S23 and S24). The MAUT scores are presented for both the parent compound and the worst transformation products (Figure $4 \mathrm{~b}$ ). DecaBDE is clearly more hazardous compared to the five alternatives regarding both PBT and PBMT if the worst transformation products are considered, while the differences among the five alternatives are minor (Table S10 and Figure 4b). Pentabromophenol (decaBDE-M3, MAUT PBMT score $=0.34)$ has the lowest MAUT score followed by PentaBDE (decaBDE-M6, MAUT PBMT score = 0.38 ), which were clearly more hazardous than any other of the studied compounds (both parents and transformation products, MAUT PBMT score $=0.51-0.73$ ). These two compounds are both much more bioaccumulative and mobile in the aquatic environment compared to their parent compound decaBDE (Table S10). PentaBDE was included as a photodegradation product of decaBDE, and based on the (a)

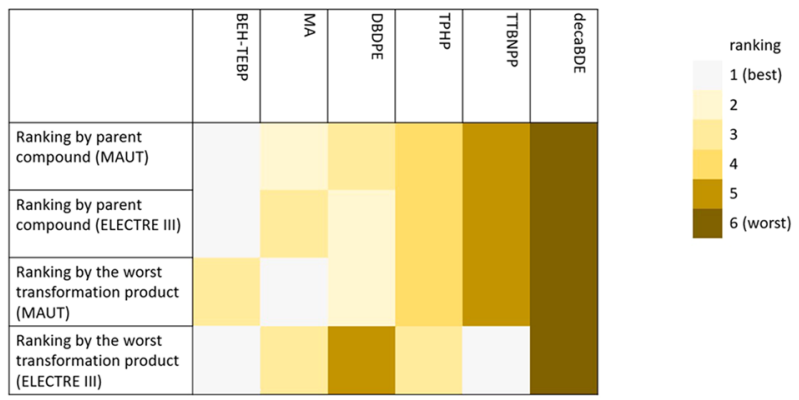

(b)

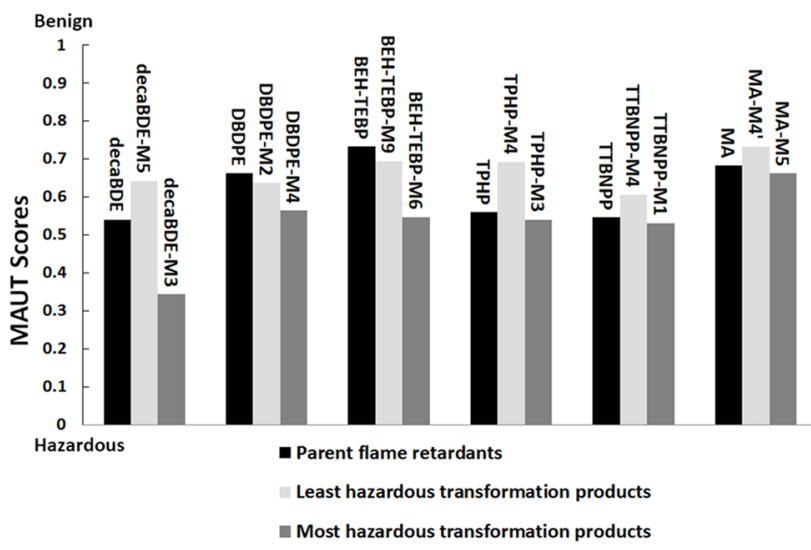

Figure 4. MAUT and ELECTRE III PBMT results with the consideration of transformation products: (a) rankings of the six flame retardants, based on parent compounds and the worst transformation products by MAUT and ELECTRE III; (b) MAUT scores of the six flame retardants (presented in the order of brominated flame retardants, organophosphates, and melamine) ranked by parent compounds, the least hazardous and the most hazardous transformation products (lower score means higher hazard).

literature, DBDPE can also photodegrade and form bromodiphenyl ethanes substituted with five to eight bromines. ${ }^{66,67}$ This suggests the importance of including photodegradation and the need to develop prediction tools to cover this pathway.

If the least hazardous transformation product is considered, BEH-TEBP, melamine, and TPHP are less hazardous concerning both PBT and PBMT, while all six parent flame retardants have similar PBMT scores (Figure $4 \mathrm{~b}$ and Table S10). Melamine is the best or second best alternative (PBT and PBMT) according to its MAUT scores, regardless of whether the ranking is done on parent compounds, or when including their transformation products.

Environmental Implications. This study presents an efficient hazard screening tool for chemical alternatives with the consideration of chemical transformations by in silico tools. The tool requires only access to (mostly) freely available in silico tools, such as those used here, and to follow the protocols presented for prioritizing predicted transformation products and conducting the PBMT assessment. As such, this approach could be automated or adapted to new in silico tools to address diverse alternatives of hazardous chemicals as part of an alternatives assessment framework. The approach to identify transformation products could be optimized in future with improved or additional models to predict transformation 
pathways or use of experimental data directly. Most of the experimental studies on transformation reactions for the substances in this study were through target chemical analysis.

There is also a need for more suspect and nontarget chemical analysis to further improve and validate in silico tools for predicting transformation products and to identify transformation products with high occurrence potential. This would form the basis to further validate, calibrate, and refine the tool presented in this study. Besides informing alternatives assessments, the tool can be used to point out potential hazardous transformation products with a high occurrence potential for environmental (bio)monitoring and screening.

In the case study, the selection of least hazardous alternatives for decaBDE was not significantly influenced by the inclusion of transformation products. It appears from this study and elsewhere that persistent chemicals are also likely to transform into similarly persistent chemicals, ${ }^{34,63}$ potentially with a higher or similar hazard level. ${ }^{63}$ The question of whether this is a general finding could have important implications for the role of persistency in risk assessments and alternatives assessments, as this essentially indicates persistent substances exhibit hazards in the environment over a longer time window than when just considering the parent compounds. For the persistence assessment, it is also possible to further develop the tool by considering a "joint persistence" of both parent compounds and key transformation products simultaneously. ${ }^{38}$ Future studies should also consider less persistent case chemicals, where the transformation products may play a more significant role in the selection of less hazardous alternatives.

Besides similar persistence, the transformation products had higher mobility in the aquatic environment compared to the parent compounds, ${ }^{34}$ which was also identified in an earlier study on pesticides as a general finding. ${ }^{63}$ This implies that groundwater transport and riverbank filtration breakthrough of transformation products can occur more quickly than for parent compounds. This further implies that the likelihood of aquatic exposure (e.g., via drinking water) and subsequent risk increases when considering both the parent and transformation products than when just considering parent substances.

By providing a reduced number of potential least hazardous alternatives, the output from this initial hazard assessment can be integrated into higher-tier alternatives assessments, such as those that combine multimedia exposure-driven and effectdriven assessment ${ }^{13,14}$ (e.g., by integrating the RAIDAR model in the case of neutral substances ${ }^{68,69}$ ) and life cycle impact assessment (e.g., using the USEtox $\operatorname{model}^{70}$ for life cycle impact assessment). Within such higher-tier models, exposure and emissions of alternatives and their transformation products over the chemical life cycle can be accounted for. For instance, here, the workflow and decision making could weigh the "mobility" relative to exposure to water over the product life cycle, and thus the importance of the PMT score output in MAUT or ELECTRE, as PMT is mainly relevant to water exposure. Similarly, the importance of photodegradation vs microbial degradation as transformation pathways could be weighed based on exposure to sunlight vs soil over the product life cycle. In many cases, the least hazardous substance may not be the best in terms of risk, if such multimedia life cycle aspects are taken into consideration. This developed tool could already be integrated in a more holistic alternatives assessment framework considering exposure, life cycle impacts, material or product performance, and cost-benefit.

\section{ASSOCIATED CONTENT}

\section{Supporting Information}

The Supporting Information is available free of charge at https://pubs.acs.org/doi/10.1021/acs.est.0c02593.

Methodology and settings for MCDA methods; chemical information for studied flame retardants and transformation products; statistical information for transformation products predicted by different in silico tools; experimental results for transformation products of case flame retardants collected from the literature and comparison with in silico predicted results; and selected QSAR models for the hazard prediction and covered hazard criteria (PDF)

Collected in silico hazard data for parent compounds and transformation products, along with MAUT and ELECTRE III calculation sheets (XLSX)

\section{AUTHOR INFORMATION}

\section{Corresponding Author}

Ziye Zheng - Department of Chemistry, Umeå University, SE90187 Umeå, Sweden; 잉 orcid.org/0000-0002-7898341X; Phone: +46 (0)76 060 3437; Email: ziye.zheng@ umu.se

\section{Authors}

Hans Peter H. Arp - Department of Environmental Engineering, Norwegian Geotechnical Institute, Ullevaal Stadion NO-0806, Oslo, Norway; Department of Chemistry, Norwegian University of Science and Technology (NTNU),

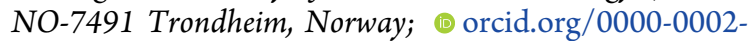
0747-8838

Gregory Peters - Division of Environmental Systems Analysis, Chalmers University of Technology, SE-412 96 Göteborg, Sweden; () orcid.org/0000-0001-8319-168X

Patrik L. Andersson - Department of Chemistry, Umeå University, SE-901 87 Umeå, Sweden; 다이.org/00000002-2088-6756

Complete contact information is available at: https://pubs.acs.org/10.1021/acs.est.0c02593

\section{Notes}

The authors declare no competing financial interest.

\section{ACKNOWLEDGMENTS}

This study was financed by a grant from the Swedish Research Council for the Environment, Agricultural Sciences and Spatial Planning (Formas) (942-2015-672). The authors acknowledge Prof. Dr. Kathrin Fenner of the Swiss Federal Institute of Aquatic Science and Technology (EAWAG) for the kind help with EAWAG-BBD/PPS, and five anonymous peer-reviewers for their constructive comments.

\section{REFERENCES}

(1) Bradley, P. M.; Chapelle, F. H. Anaerobic Mineralization of Vinyl Chloride in $\mathrm{Fe}(\mathrm{III})$-Reducing, Aquifer Sediments. Environ. Sci. Technol. 1996, 30, 2084-2086.

(2) Cui, N.; Zhang, X.; Xie, Q.; Wang, S.; Chen, J.; Huang, L.; Qiao, X.; Li, X.; Cai, X. Toxicity profile of labile preservative bronopol in water: the role of more persistent and toxic transformation products. Environ. Pollut. 2011, 159, 609-615.

(3) Donner, E.; Kosjek, T.; Qualmann, S.; Kusk, K. O.; Heath, E.; Revitt, D. M.; Ledin, A.; Andersen, H. R. Ecotoxicity of 
carbamazepine and its UV photolysis transformation products. Sci. Total Environ. 2013, 443, 870-876.

(4) ECHA. Guidance on Information Requirements and Chemical Safety Assessment; Chapter R. 11: PBT/vPvB Assessment, 2017.

(5) Van Zelm, R.; Huijbregts, M. A.; van de Meent, D. Transformation products in the life cycle impact assessment of chemicals. Environ. Sci. Technol. 2010, 44, 1004-1009.

(6) Howard, P. H.; Muir, D. C. Identifying new persistent and bioaccumulative organics among chemicals in commerce. III: Byproducts, impurities, and transformation products. Environ. Sci. Technol. 2013, 47, 5259-5266.

(7) Pakalin, S.; Cole, T.; Steinkellner, J.; Nicolas, R.; Tissier, C.; Munn, S.; Eisenreich, S. Review on Production Processes of Decabromodiphenyl Ether (DECABDE) Used in Polymeric Applications in Electrical and Electronic Equipment, and Assessment of the Availability of Potential Alternatives to DECABDE; European Commission, Directorate General Joint Research Center, European Chemicals Bureau, 2007.

(8) ECHA Registered Substances of the REACH Registration Dossiers, Bis(pentabromophenyl) Ether, PBT Assessment. https:// echa.europa.eu/registration-dossier/-/registered-dossier/14217/2/3.

(9) Report of the Persistent Organic Pollutants Review Committee on the work of its fifth meeting, addendum: General guidance on considerations related to alternativesand substitutes for POPs; Persistent Organic Pollutants Review Committee, 2009.

(10) A Framework to Guide Selection of Chemical Alternatives; National Academies Press: Washington, DC, 2014.

(11) Clean Production Action. Green Screen Transformation Product Worksheet \& Resources. 2018, https://www. greenscreenchemicals.org/images/ee_images/uploads/resources/ GreeScreen1.4-Annex9-1.18.pdf.

(12) Lavoie, E. T.; Heine, L. G.; Holder, H.; Rossi, M. S.; Lee, R. E.; Connor, E. A.; Vrabel, M. A.; DiFiore, D. M.; Davies, C. L. Chemical Alternatives Assessment: Enabling Substitution to Safer Chemicals. Environ. Sci. Technol. 2010, 44, 9244-9249.

(13) Martin, T. M. A framework for an alternatives assessment dashboard for evaluating chemical alternatives applied to flame retardants for electronic applications. Clean Technol. Environ. Policy 2017, 19, 1067-1086.

(14) Escher, B. I.; Fenner, K. Recent advances in environmental risk assessment of transformation products. Environ. Sci. Technol. 2011, 45, 3835-3847.

(15) Dimitrov, S.; Diderich, R.; Sobanski, T.; Pavlov, T.; Chankov, G.; Chapkanov, A.; Karakolev, Y.; Temelkov, S.; Vasilev, R.; Gerova, K.; et al. QSAR Toolbox-Workflow and major functionalities. SAR QSAR Environ. Res. 2016, 27, 203-219.

(16) Wolfe, K.; Pope, N.; Parmar, R.; Galvin, M.; Stevens, C.; Weber, E.; Flaishans, J.; Purucker, T. In Chemical transformation system: cloud based cheminformatic services to support integrated environmental modeling, Proceedings of the iEMSs 2016, Toulouse, France, July 10-14, 2016.

(17) CTS: Chemical Transformation Simulator. https://qed.epacdx. net/cts/.

(18) Cruciani, G.; Carosati, E.; De Boeck, B.; Ethirajulu, K.; Mackie, C.; Howe, T.; Vianello, R. MetaSite: Understanding Metabolism in Human Cytochromes from the Perspective of the Chemist. J. Med. Chem. 2005, 48, 6970-6979.

(19) Stardrop; Optibrium Ltd.: Cambridge, U.K..

(20) Ott, M. A.; Toy, R. L.; Button, W. G. An Approach toward the Prediction of Chemical Degradation Pathways; Lhasa Limited, pp 2223.

(21) Marchant, C. A.; Briggs, K. A.; Long, A. In silico tools for sharing data and knowledge on toxicity and metabolism: Derek for windows, meteor, and vitic. Toxicol. Mech. Methods 2008, 18, 177187.

(22) Djoumbou-Feunang, Y.; Fiamoncini, J.; Gil-de-la-Fuente, A.; Greiner, R.; Manach, C.; Wishart, D. S. BioTransformer: a comprehensive computational tool for small molecule metabolism prediction and metabolite identification. J. Cheminform. 2019, 11, 2.
(23) Gao, J.; Ellis, L. B. M.; Wackett, L. P. The University of Minnesota Biocatalysis/Biodegradation Database: improving public access. Nucleic Acids Res. 2010, 38, D488-D491.

(24) Greggs, W.; Burns, T.; Egeghy, P.; Embry, M. R.; Fantke, P.; Gaborek, B.; Heine, L.; Jolliet, O.; Lee, C.; Muir, D. Qualitative approach to comparative exposure in alternatives assessment. Integr. Environ. Assess. Manage. 2018, 15, 880-894.

(25) Beaudrie, C.; Corbett, C. J.; Lewandowski, T. A.; Malloy, T.; Zhou, X. Evaluating the Application of Decision Analysis Methods in Simulated Alternatives Assessment Case Studies: Potential Benefits and Challenges of Using MCDA. Integr. Environ. Assess. Manage. 2020, 17, 27-41.

(26) Zheng, Z.; Peters, G. M.; Arp, H. P. H.; Andersson, P. L. Combining in Silico Tools with Multicriteria Analysis for Alternatives Assessment of Hazardous Chemicals: A Case Study of Decabromodiphenyl Ether Alternatives. Environ. Sci. Technol. 2019, 53, 63416351.

(27) Estimation Programs Interface Suite for Microsoft Windows, v 4.11; United States Environmental Protection Agency: Washington, DC, 2012.

(28) Benfenati, E.; Manganaro, A.; Gini, G. C. In VEGA-QSAR: AI Inside a Platform for Predictive Toxicology, Proceedings of Workshop PAI: Turin, Italy, 2013.

(29) Martin, T. Toxicity Estimation Software Tool (TEST); U.S. Environmental Protection Agency: Washington, DC, 2016.

(30) Sushko, I.; Novotarskyi, S.; Körner, R.; Pandey, A. K.; Rupp, M.; Teetz, W.; Brandmaier, S.; Abdelaziz, A.; Prokopenko, V. V.; Tanchuk, V. Y.; Todeschini, R.; Varnek, A.; Marcou, G.; Ertl, P.; Potemkin, V.; Grishina, M.; Gasteiger, J.; Schwab, C.; Baskin, I. I.; Palyulin, V. A.; Radchenko, E. V.; Welsh, W. J.; Kholodovych, V.; Chekmarev, D.; Cherkasov, A.; Aires-de-Sousa, J.; Zhang, Q.-Y.; Bender, A.; Nigsch, F.; Patiny, L.; Williams, A.; Tkachenko, V.; Tetko, I. V. Online chemical modeling environment (OCHEM): web platform for data storage, model development and publishing of chemical information. J. Comput.-Aided Mol. Des. 2011, 25, 533-554.

(31) Mansouri, K.; Abdelaziz, A.; Rybacka, A.; Roncaglioni, A.; Tropsha, A.; Varnek, A.; Zakharov, A.; Worth, A.; Richard, A. M.; Grulke, C. M.; et al. CERAPP: Collaborative estrogen receptor activity prediction project. Environ. Health Perspect. 2016, 124, 1023.

(32) Mansouri, K.; Kleinstreuer, N.; Abdelaziz, A. M.; Alberga, D.; Alves, V. M.; Andersson, P. L.; Andrade, C. H.; Bai, F.; Balabin, I.; Ballabio, D.; et al. CoMPARA: Collaborative Modeling Project for Androgen Receptor Activity. Environ. Health Perspect. 2020, 128, No. 027002.

(33) Rybacka, A.; Rudén, C.; Tetko, I. V.; Andersson, P. L. Identifying potential endocrine disruptors among industrial chemicals and their metabolites-development and evaluation of in silico tools. Chemosphere 2015, 139, 372-378.

(34) Arp, H.; Brown, T.; Berger, U.; Hale, S. Ranking REACH registered neutral, ionizable and ionic organic chemicals based on their aquatic persistency and mobility. Environ. Sci.: Processes Impacts 2017, 19, 939-955.

(35) Zhang, X.; Brown, T. N.; Wania, F.; Heimstad, E. S.; Goss, K. $\mathrm{U}$. Assessment of chemical screening outcomes based on different partitioning property estimation methods. Environ. Int. 2010, 36, 514-520.

(36) Muir, D.; Zhang, X.; de Wit, C. A.; Vorkamp, K.; Wilson, S. Identifying further chemicals of emerging arctic concern based on 'in silico'screening of chemical inventories. Emerging Contam. 2019, 5, 201-210.

(37) Sedykh, A.; Saiakhov, R.; Klopman, G. META V. A model of photodegradation for the prediction of photoproducts of chemicals under natural-like conditions. Chemosphere 2001, 45, 971-981.

(38) Ng, C. A.; Scheringer, M.; Fenner, K.; Hungerbuhler, K. A Framework for Evaluating the Contribution of Transformation Products to Chemical Persistence in the Environment. Environ. Sci. Technol. 2011, 45, 111-117. 
(39) Fenner, K.; Scheringer, M.; Hungerbühler, K. Joint persistence of transformation products in chemicals assessment: case studies and uncertainty analysis. Risk Anal. 2003, 23, 35-53.

(40) Fenner, K.; Schenker, U.; Scheringer, M. Modelling Environmental Exposure to Transformation Products of Organic Chemicals. Transformation Products of Synthetic Chemicals in the Environment, 2008; pp 121-149.

(41) Wicker, J.; Fenner, K.; Ellis, L.; Wackett, L.; Kramer, S. Predicting biodegradation products and pathways: a hybrid knowledge-and machine learning-based approach. Bioinformatics 2010, 26, 814-821.

(42) Shelton, D. R.; Karns, J. S.; McCarty, G. W.; Durham, D. R. Metabolism of Melamine by Klebsiella terragena. Appl. Environ. Microbiol. 1997, 63, 2832-2835.

(43) Tisler, S.; Zindler, F.; Freeling, F.; Nödler, K.; Toelgyesi, L.; Braunbeck, T.; Zwiener, C. Transformation Products of Fluoxetine Formed by Photodegradation in Water and Biodegradation in Zebrafish Embryos (Danio rerio). Environ. Sci. Technol. 2019, 53, $7400-7409$.

(44) Marchant, C. A.; Rosser, E. M.; Vessey, J. D. A k-nearest neighbours approach using metabolism-related fingerprints to improve in silico metabolite ranking. Mol. Inf. 2017, 36, No. 1600105.

(45) Strempel, S.; Scheringer, M.; Ng, C. A.; Hungerbühler, K. Screening for PBT chemicals among the "existing" and "new" chemicals of the EU. Environ. Sci. Technol. 2012, 46, 5680-5687.

(46) Sandholm, A.; Emanuelsson, B.-M.; Wehler, E. K. Bioavailability and half-life of decabromodiphenyl ether (BDE-209) in rat. Xenobiotica 2003, 33, 1149-1158.

(47) Van der Ven, L. T.; van de Kuil, T.; Leonards, P. E.; Slob, W.; Cantón, R. F.; Germer, S.; Visser, T. J.; Litens, S.; Håkansson, H.; Schrenk, D.; et al. A 28-day oral dose toxicity study in Wistar rats enhanced to detect endocrine effects of decabromodiphenyl ether (decaBDE). Toxicol. Lett. 2008, 179, 6-14.

(48) Mörck, A.; Hakk, H.; Örn, U.; Wehler, E. K. Decabromodiphenyl ether in the rat: absorption, distribution, metabolism, and excretion. Drug Metab. Dispos. 2003, 31, 900-907.

(49) Deng, D.; Liu, J.; Xu, M.; Zheng, G.; Guo, J.; Sun, G. Uptake, translocation and metabolism of decabromodiphenyl ether (BDE209) in seven aquatic plants. Chemosphere 2016, 152, 360-368.

(50) Draft Screening Assessment of Certain Organic Flame Retardants Substance Grouping: 1,3,5-Triazine-2,4,6-triamine (Melamine); Environment and Climate Change Canada, Health Canada: Canada, 2016.

(51) Crews, G. M.; Ripperger, W.; Kersebohm, D. B.; Güthner, T.; Mertschenk, B. Melamine and guanamines. Ullmann's Encyclopedia of Industrial Chemistry; Wiley, 2000.

(52) Bai, X.-y.; Lu, S.-y.; Xie, L.; Zhang, B.; Song, S.-m.; He, Y.; Ouyang, J.-p.; Zhang, T. A pilot study of metabolites of organophosphorus flame retardants in paired maternal urine and amniotic fluid samples: potential exposure risks of tributyl phosphate to pregnant women. Environ. Sci.: Processes Impacts 2019, 21, 124-132.

(53) Zhang, B.; Lu, S.; Huang, M.; Zhou, M.; Zhou, Z.; Zheng, H.; Jiang, Y.; Bai, X.; Zhang, T. Urinary metabolites of organophosphate flame retardants in 0-5-year-old children: Potential exposure risk for inpatients and home-stay infants. Environ. Pollut. 2018, 243, 318-325.

(54) Carignan, C. C.; Mínguez-Alarcón, L.; Butt, C. M.; Williams, P. L.; Meeker, J. D.; Stapleton, H. M.; Toth, T. L.; Ford, J. B.; Hauser, R.; Team, E. S. Urinary concentrations of organophosphate flame retardant metabolites and pregnancy outcomes among women undergoing in vitro fertilization. Environ. Health Perspect. 2017, 125, No. 087018.

(55) Wang, G.; Du, Z.; Chen, H.; Su, Y.; Gao, S.; Mao, L. Tissuespecific accumulation, depuration, and transformation of triphenyl phosphate (TPHP) in adult zebrafish (Danio rerio). Environ. Sci. Technol. 2016, 50, 13555-13564.

(56) Sasaki, K.; Suzuki, T.; Takeda, M.; Uchiyama, M. Metabolism of phosphoric acid triesters by rat liver homogenate. Bull. Environ. Contam. Toxicol. 1984, 33, 281-288.

(57) Springer, C.; Dere, E.; Hall, S. J.; McDonnell, E. V.; Roberts, S. C.; Butt, C. M.; Stapleton, H. M.; Watkins, D. J.; McClean, M. D.;
Webster, T. F.; et al. Rodent thyroid, liver, and fetal testis toxicity of the monoester metabolite of bis-(2-ethylhexyl) tetrabromophthalate (TBPH), a novel brominated flame retardant present in indoor dust. Environ. Health Perspect. 2012, 120, 1711-1719.

(58) Fenner, K.; Gao, J.; Kramer, S.; Ellis, L.; Wackett, L. Datadriven extraction of relative reasoning rules to limit combinatorial explosion in biodegradation pathway prediction. Bioinformatics 2008, 24, 2079-2085.

(59) Kajiwara, N.; Noma, Y.; Takigami, H. Photolysis studies of technical decabromodiphenyl ether (DecaBDE) and ethane (DeBDethane) in plastics under natural sunlight. Environ. Sci. Technol. 2008, 42, 4404-4409.

(60) Ecological State of the Science Report on Decabromodiphenyl Ether (decaBDE); Bioaccumulation and Transformation: Environment Canada, 2010.

(61) Mas, S.; de Juan, A.; Lacorte, S.; Tauler, R. Photodegradation study of decabromodiphenyl ether by UV spectrophotometry and a hybrid hard-and soft-modelling approach. Anal. Chim. Acta 2008, 618, $18-28$.

(62) Söderström, G.; Sellström, U.; de Wit, C. A.; Tysklind, M. Photolytic debromination of decabromodiphenyl ether (BDE 209). Environ. Sci. Technol. 2004, 38, 127-132.

(63) Boxall, A. B.; Sinclair, C. J.; Fenner, K.; Kolpin, D.; Maund, S. J. Peer Reviewed: When Synthetic Chemicals Degrade in the Environment. Environ. Sci. Technol. 2004, 38, 368A-375A.

(64) Arp, H. P. H.; Hale, S. E. REACH: Improvement of guidance methods for the identification and evaluation of PM/PMT substances. UBA TEXTE 126/2019. German Environment Agency (UBA), Dessau-Roßlau, Germany. p 130, ISBN 1862-4804.

(65) Jang, Y. H.; Hwang, S.; Chang, S. B.; Ku, J.; Chung, D. S. Acid dissociation constants of melamine derivatives from density functional theory calculations. J. Phys. Chem. A 2009, 113, 13036-13040.

(66) Wang, J.; Chen, S.; Nie, X.; Tian, M.; Luo, X.; An, T.; Mai, B. Photolytic degradation of decabromodiphenyl ethane (DBDPE). Chemosphere 2012, 89, 844-849.

(67) Klimm, A.; Brenner, D.; Lok, B.; Sprengel, J.; Krätschmer, K.; Vetter, W. Photolytic transformation products of decabromodiphenyl ethane (DBDPE). Environ. Sci. Technol. 2019, 53, 6302-6309.

(68) Arnot, J. A.; MacKay, D.; Webster, E.; Southwood, J. M. Screening level risk assessment model for chemical fate and effects in the environment. Environ. Sci. Technol. 2006, 40, 2316-2323.

(69) Arnot, J. A.; Brown, T. N.; Wania, F.; Breivik, K.; McLachlan, M. S. Prioritizing chemicals and data requirements for screening-level exposure and risk assessment. Environ. Health Perspect. 2012, 120, $1565-1570$.

(70) Rosenbaum, R. K.; Bachmann, T. M.; Gold, L. S.; Huijbregts, M. A.; Jolliet, O.; Juraske, R.; Koehler, A.; Larsen, H. F.; MacLeod, M.; Margni, M.; et al. USEtox-the UNEP-SETAC toxicity model: recommended characterisation factors for human toxicity and freshwater ecotoxicity in life cycle impact assessment. Int. J. Life Cycle Assess. 2008, 13, 532. 